\title{
Lensless inline digital holography versus Fourier ptychography: phase estimation of a large transparent bead
}

\author{
Hongyu Li, Anastasia Bozhok, Yoshitate Takakura,* Jean Dellinger, \\ Patrice Twardowski, and Jihad Zallat \\ University of Strasbourg, ICube-UMR 7357 CNRS, Pôle API, Illkirch Cedex, France
}

\begin{abstract}
Lensless inline digital holographic microscopy (LI-DHM) and Fourier ptychographic microscopy (FPM) are two widespread quantitative phase imaging (QPI) techniques. They have been employed in various fields, especially for biological slice imaging because of their simplicity in use, stability in structure, and also large field of view. Spherical phase response (for example from HeLa cells) is commonly observed in biological imagery. As a consequence, for calibration and validation purposes, small (several to tenth of microns in diameter) transparent microbeads have been used as standards. Phase imaging of their large counterparts (hundreds of microns in diameter) using either LI-DHM or FPM has not been reported so far. We are aiming to analyze the phase response of a $146-\mu \mathrm{m}$ soda-lime microsphere. It has been immersed in Canada balsam to reduce phase difference and to avoid overexposed diffraction rings. The phase estimation issue has been tackled using approaches that involve either Gerchberg-Saxton type algorithms or an inverse problem-based procedure. Confronting the results confirms the QPI capability for both imaging techniques to assess phase responses from such a large transparent object. (C) 2020 Society of Photo-Optical Instrumentation Engineers (SPIE) [DOI: 10.1117/1.OE.59.8.083104]
\end{abstract}

Keywords: lensless inline digital holography; Fourier ptychography; quantitative phase imaging; phase retrieval; microsphere.

Paper 20200504 received Apr. 30, 2020; accepted for publication Jul. 28, 2020; published online Aug. 17, 2020.

\section{Introduction}

Retrieving quantitative phase information from a transparent object is an essential and important issue for biological tissue imaging. In the past decades, a number of quantitative phase imaging (QPI) techniques have been introduced. ${ }^{1}$ With the development of computational imaging, QPI with simple optical setups can be achieved and is still rapidly expanding. For example, the transport-of-intensity equation technique ${ }^{2}$ is an approach that allows the retrieval of phases from images in situations where the illuminations are noncoherent and nonuniform. In cases where the lightings are coherent or almost monochromatic, lensless inline digital holographic microscopy (LI-DHM) and Fourier ptychographic microscopy (FPM) ${ }^{3}$ are two representative techniques that allow high-resolution phase imaging with basic setups.

Inline holography was first introduced by Gabor ${ }^{4}$ in 1948. Due to the twin image distortion, this technique was neglected for a long time. After 2000, due to the improvement of computational imaging techniques along with the development of digital cameras, retrieval of phase information and reduction of the twin image effect have been made possible. ${ }^{5,6}$ Owing to its simplicity, LI-DHM is capable of accomplishing microscopic imaging tasks under extreme environments such as undersea microorganism microscopic imaging. ${ }^{7}$ Recently, LI-DHMs have become popular. With samples positioned as close as possible to the camera, the demand for light coherency may be reduced. With magnification nearly equal to 1 , a high field of view (FOV) that embraces the sensor can be obtained. ${ }^{8,9}$ Also, subpixel super-resolution techniques have been applied to improve performances. ${ }^{8,10,11}$ Rigid and compact microscopes have been

*Address all correspondence to Yoshitate Takakura, E-mail: y.takakura@unistra.fr 
built with those on chip LI-DHMs. ${ }^{12,13}$ They are cost effective and at the same time maintain performances comparable with conventional QPI microscopes. ${ }^{14,15}$

FPM was introduced in 2013 by Zheng et al. ${ }^{3}$ By recording and combining images with different angular illuminations, it can achieve high resolution using a low numerical aperture (NA) microscope objective. At the same time, it has the large FOV of the low NA lens. Shortly after the first publication, phase imaging capability of the FPM has been validated. ${ }^{16}$ In the most widely used setup, the angular scanning illumination is produced by a matrix of light-emitting diodes (LEDs), which is inexpensive and easy to integrate into a conventional optical microscope. Due to the simplicity of implementation and the remarkable imaging performance of the technique, many applications in the field of biological slide imaging have been explored in recent years. ${ }^{17-20}$ For example, HeLa cell mitosis has been observed in vitro with a 388-nm half-pitch resolution. ${ }^{21}$

To the best of our knowledge, there seems to be no report on a quantitative analysis of QPI capability of LI-DHM and FPM for relatively large transparent objects (more than a hundred of microns). Due to the similarity of the optical setups, it could be valuable to assess the performances of both techniques.

The large phase object used in this work is a $146-\mu \mathrm{m}$-diameter soda-lime microsphere. It is immersed in Canada balsam to reduce phase difference to avoid overexposed diffraction rings. The diameter of the microsphere has been inferred with a conventional phase-contrast microscope and the nonquantitative phase images served as basic references for further analyses. The phase response of the sample has been estimated by LI-DHM and confronted with the results from FPM.

\section{Sample and Methods}

In this section, basic parameters of the microsphere sample are introduced. LI-DHM and FPM setups along with the corresponding phase estimation methods are presented.

\subsection{Transparent Phase Sample}

For QPI validation purpose, several objects have been considered as standards: red blood cells, ${ }^{12,16}$ laser lines etched on glass, ${ }^{12}$ commercial phase standards, ${ }^{22}$ and samples with known refractive index such as optical fibers ${ }^{23}$ or polystyrene microbeads ${ }^{16}$ immersed in oil. We chose to fabricate our own standard by isolating a single soda-lime (refractive index $n_{\text {glass }}=1.522$ at $633 \mathrm{~nm}$ ) microsphere on a slide, immersed in Canada balsam for immobilization. The diameter provided by the manufacturer is $150 \mu \mathrm{m}$ with a standard deviation-to-mean ratio of $10 \%$. The exact diameter of the sphere is then inferred from images captured with a phase-contrast microscope as shown in Fig. 1(b). To set the focus plane at the middle of the sample, we have analyzed the captured images at a vicinity of the microsphere boundary and maximized the sharpness of the observed arc. The estimated diameter is $146 \mu \mathrm{m}$. The refractive index of the Canada balsam has been measured separately using a refractometer. Depending on the experiments, variations

(a)

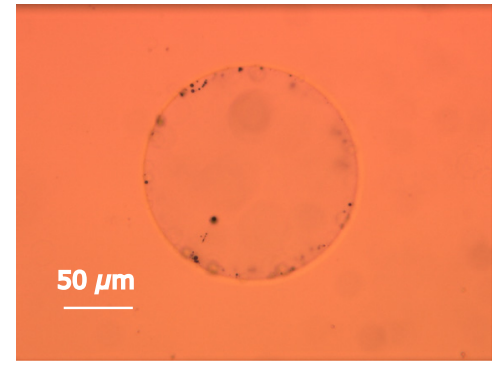

(b)

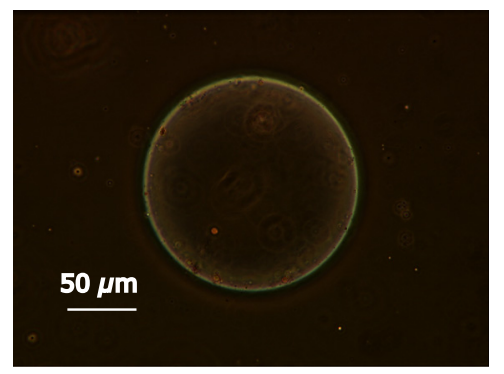

Fig. 1 Images of microsphere from conventional microscope. Diameter of the sphere is $146 \mu \mathrm{m}$. Image under (a) bright-field microscope and (b) phase-contrast microscope. 
ranging from 1.523 to 1.533 have been observed in the red band. For that reason, we have chosen to compare phase responses retrieved by each of our instruments.

\subsection{Lensless Inline Digital Holographic Microscope}

\subsubsection{Image formation model}

The schematic of Gabor LI-DHM is shown in Fig. 2. The interference pattern recorded on a hologram is proportional to $H(x, y)$ as given by Eq. (1):

$$
H(x, y)=|R(x, y)|^{2}+|O(x, y)|^{2}+R^{*}(x, y) O(x, y)+R(x, y) O^{*}(x, y),
$$

where $R(x, y)$ is the reference wave and $O(x, y)$ represents the object wave. The first term in Eq. (1) corresponds to the background. It may be subtracted from $H(x, y)$. In cases where phase only objects are considered, the second term may be neglected compared with the reference wave. ${ }^{24}$ The last ones carry informations to be retrieved. In the LI-DHM configuration where a point source is used, the waves diffracted by the object are captured in $O(x, y)$. The undiffracted light will serve as the reference $R(x, y)$. Because of the well-known twin effect, a phase image cannot be recovered directly from an inline hologram. Additional steps are necessary.

\subsubsection{Experimental setup}

In this work, we chose to use the classical Gabor's configuration. ${ }^{25} \mathrm{~A} 632.8-\mathrm{nm}$ polarized $\mathrm{He}-\mathrm{Ne}$ laser is focused by a microscope objective $(\mathrm{NA}=0.35)$ onto a $25-\mu \mathrm{m}$-diameter pinhole. The filtered beam serves as the point source for the LI-DHM setup. The distance between the point source and the sensor is estimated numerically to be about $21.775 \mathrm{~cm}$. The sensor is an 8-bit monochrome CMOS camera with $2048 \times 2048$ pixels of size $7.4 \mu \mathrm{m}$ each. The object and the sensor are set to be about $18 \mathrm{~cm}$ away from each other so that magnification is about 5.5. The system is shown in Fig. 2. The NA of a LI-DHM can be computed by means of the formula ${ }^{26} \mathrm{NA}=(W / 2)\left[(W / 2)^{2}+z^{2}\right]^{-1 / 2}$, where $W$ is the width of the sensor and $z$ is the distance between the object plane and the hologram plane. For the current setup, the obtained value is $\mathrm{NA} \approx 0.041$. With the resolution and depth of field (DOF) provided by $(2 \delta x)=$ $\lambda /(\mathrm{NA})$ and $(2 \delta z)=\lambda /(\mathrm{NA})^{2}$, respectively, ${ }^{27}$ those features are equal to $(2 \delta x)=15.4 \mu \mathrm{m}$ and $(2 \delta z)=377 \mu \mathrm{m}$. Therefore, it is worth mentioning that the DOF is larger than the diameter of the sample.
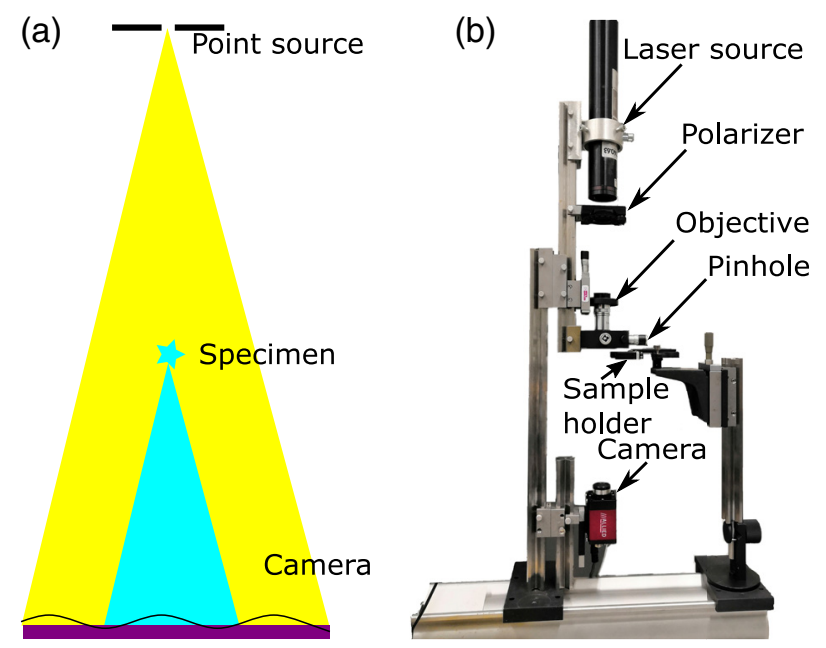

Fig. 2 Gabor LI-DHM built for this work: (a) schematic diagram and (b) experimental setup. 


\subsubsection{Reconstruction}

Two well-established methods were employed for phase estimation. The first one lies on the commonly used Gerchberg-Saxton (G-S) type iterative phase retrieval method. ${ }^{28}$ The second one is based on an inverse problem approach. ${ }^{29,30}$

The procedure involving G-S projection includes two steps. To start with, an autofocusing algorithm is applied to get the right focusing position. To that end, the sparsity of gradient $(\mathrm{SOG})^{31}$ method has been employed. The idea is to look for the location where the gradient of the reconstructed wavefront is the sparsest. It can be applied to both amplitude and phase objects. While the Tamura coefficient (TC) or the Gini index can be used both to evaluate sparsity, ${ }^{30} \mathrm{TC}$ has been selected for its robustness. ${ }^{32}$ Its expression is provided by Eq. (2):

$$
\mathrm{TC}(g)=\sqrt{\frac{\delta(|g|)}{\langle|g|\rangle}},
$$

where $\delta$ stands for the standard deviation, $\langle\cdot\rangle$ is the ensemble average, and $g$ represents the gradient of the reconstructed wavefront.

Once the focus has been estimated, the G-S algorithm is launched and coupled with the socalled positive absorption constraint. ${ }^{6}$ The latter consists of imposing the limit of 1 to all pixel values that surpass 1 in the two-dimensional (2-D) transmittance that approximates the object. Since the size of the microsphere is known, an object support may be introduced to accelerate the convergence of the algorithm. ${ }^{33,34}$ Also, it is worth mentioning that the point-source illuminated inline hologram may be reconstructed as a plane-wave illuminated hologram with an equivalent propagation distance and an additional magnification rate. ${ }^{35-37}$ As a consequence, propagation between the object plane and the hologram plane may be computed with a plane-wave illumination. The real distance $z_{s p h}$ between the object and the camera, and the equivalent propagation distance $z_{p l}$ are related according to Eq. (3):

$$
\frac{1}{z_{p l}}=\frac{1}{z_{s p h}}-\frac{1}{d},
$$

where $d$ is the distance between the point source and the camera. Here, $z_{p l}$ is about $1 \mathrm{~m}$. The band-limited angular spectrum method (ASM) algorithm ${ }^{38}$ has been used for calculating propagation between the object plane and the hologram plane. Aliasing when using classical ASM in the far field could then be avoided.

As for the second investigation, a parameter-based inverse problem algorithm has been used since the regular geometrical shape of the microsphere involves very few variables. ${ }^{29}$ It is more robust than the general approach that consists of reconstructing the transmittance distribution directly. ${ }^{39}$ It was introduced by Soulez et al. ${ }^{40}$ to track the traces of particles and to estimate their size. The detailed mathematical development can be found in the dissertation of Denis. ${ }^{41} \mathrm{We}$ have adapted the approach to our problem. It will be briefly summarized here. The cost function can be described in Eq. (4):

$$
\ell(\vec{p}, \alpha, \beta)=\sum_{x} \sum_{y} W(x, y) \cdot\left[I_{H}(x, y)-\alpha I_{\text {simu }}(\vec{p} ; x, y)+\beta\right]^{2},
$$

where $I_{H}$ is the hologram captured experimentally and $I_{\text {simu }}$ is the simulated diffraction pattern. $W$ represents a weight mask $W(x, y)=1 / \operatorname{var}\left[I_{H}(x, y)\right]$ that defines a matrix of inverse variances for each pixel. For elements that are not taken into account (pixels outside the hologram, dead pixels), $W(x, y)$ is set to zero. ${ }^{29} \alpha$ and $\beta$ are the two real positive parameters with $\alpha$ related to the energy received by the camera and $\beta$ the DC component of the hologram (reference wave intensity). ${ }^{41}$ The expressions of $\alpha^{*}$ and $\beta^{*}$ that minimize $\ell(\vec{p}, \alpha, \beta)$ may be obtained analytically. Detailed calculations that permit one to exhibit the cost function is provided in Appendix A. The unknown $\vec{p}=\left(x_{p}, y_{p}, z_{p}, D_{p}\right)$ is formed by the position of the center and the diameter of a microsphere. For our case, the diameter $D_{p}$ is already known. As the microsphere is far enough from the point source $(\approx 3.9 \mathrm{~cm})$ and the system depth of field is larger than its diameter, 
the sample's response can be approximated by a complex transmittance for incident waves that are almost plane. ${ }^{42}$ The phase distribution $P(r)$ across the sample may be obtained by evaluating the delay undertaken by each directly transmitted ray and can be described by Eq. (5):

$$
P(r)=P_{\max } \sqrt{1-\left(\frac{r}{R}\right)^{2}} \quad r<R=0 \quad \text { otherwise, }
$$

with $r=\sqrt{\left(x^{2}+y^{2}\right)} . R$ is the radius of the sphere, and $P_{\max }$ identifies the maximum phase shift that is produced by the difference of refractive indices between glass and optical glue. Since a unique sphere is present in our case, its lateral position $\left(x_{p}, y_{p}\right)$ may be determined prior to the current optimization process. We then have to seek $\vec{p}=\left(z_{p}, P_{\max }\right)$. With the object plane far enough from the camera, the sample may be considered as a phase disk that follows Eq. (5). Unfortunately, diffraction pattern of a phase disk illuminated by spherical waves cannot be calculated analytically, unlike objects in Ref. 41. As an alternative, a fast Fourier transform (FFT)based Fresnel diffraction method will be used to obtain $I_{\text {simu }}$. It is worth reminding that the latter, being the simulated diffraction pattern, is computed by propagating the complex transmittance that approximates the object onto the hologram plane. Finally, optimization is achieved by means of a sequential quadratic programming algorithm. ${ }^{43}$

\subsection{Fourier Ptychographic Microscope}

\subsubsection{Image formation model}

FPM was chosen to confirm the phase distribution obtained by the holographic system. It is a computational imaging technique based on Fourier optics. Unlike digital holography where interference patterns are recorded, FPM captures intensity data in the spatial domain. Both methods necessitate phase retrieval algorithms. Ptychography has another key advantage in addition to its QPI capabilities, namely, the improvement of the system's resolution.

The limits of imaging performance in optical microscopy are mainly imposed by the diffraction phenomenon, which can be quantified by the NA of the system and the wavelength of the illumination. Indeed, in the context of microscopy, this phenomenon can be explained by examining the Fourier spectrum of the light that has passed through the sample and the system. More precisely, it is the pupil of the microscope objective that acts as a low-pass filter, cutting off the high frequencies of the light field, thus limiting the resulting resolution. The degree to which the frequencies are cut off depends on the radius of the pupil function, which in turn depends on the NA. The main idea of Fourier ptychography is to synthetically enlarge the NA of the system by capturing images corresponding to different parts of the spectrum and then by assembling them in the Fourier domain.

By placing a coherent light source sufficiently far below the sample, the illumination can be approximated by a plane wave described mathematically by a complex exponential. When such a source is moved away from the optical axis, the wave arrives at the sample with an oblique incidence. Such an interaction can be modeled as a multiplication with the transmission function of the sample $o(x, y)$ in the spatial domain, which results in a shift of the spectrum in the Fourier domain. Therefore, the conventional optical configuration of the FPM involves an array of LEDs used to produce a set of different illumination angles. The imaging model for the perfect noisefree case is given by Eq. (6):

$$
I_{l}(x, y)=\left|\mathcal{F T}^{-1}\left\{P\left(k_{x}, k_{y}\right) O\left(k_{x}-k_{x_{l}}, k_{y}-k_{y_{l}}\right)\right\}\right|^{2}, \quad l=1, \ldots, L,
$$

where $I_{l}(x, y)$ is an intensity image captured for each LED $l ; \mathcal{F} \mathcal{T}^{-1}$ is the inverse 2-D FT operator; $P\left(k_{x}, k_{y}\right)$ is the pupil function of the lens; $\left(k_{x_{l}}, k_{y_{l}}\right)$ is the spatial frequency shift for the $l$ 'th LED; $O\left(k_{x}, k_{y}\right)$ is the spectrum of the sample's transmission function $o(x, y)$.

In real experimental conditions, the model described by Eq. (6) is only an approximation of the real physical process and the data set $I_{l}(x, y), l=1, \ldots, L$ is corrupted by noise. 


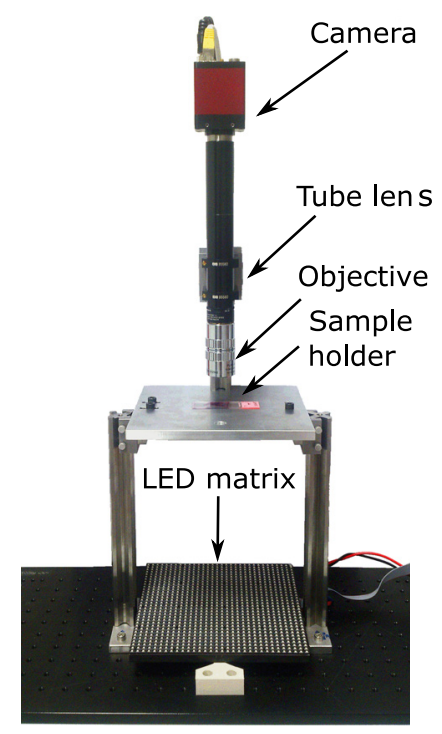

Fig. 3 Experimental setup of FPM. It consists of combining a digital camera, a low NA microscope objective, and a LED array.

\subsubsection{Experimental setup}

The optical configuration built from scratch and its components are shown in Fig. 3. The camera used is equipped with a sensor of $2048 \times 2048$ elements, a pixel size of $6.5 \mu \mathrm{m} \times 6.5 \mu \mathrm{m}$, and a 16-bit converter. The objective is a $2 \times$ Edmund Optics infinity-corrected coupled with a $f=$ $200 \mathrm{~mm}$ lens tube to focus the image on the camera sensor. The experimentally determined NA is 0.07 . Finally, the LED matrix is a red-green-blue $32 \times 32$ panel with $6-\mathrm{mm}$ spacing supplied by Adafruit. The position of the specimen holder, as measured by a ruler, gives a length of $245 \mathrm{~mm}$ between the object and the LED array.

For ongoing investigations, $20 \times 20$ grayscale images are captured using illumination of wavelength $\lambda=0.630 \mu \mathrm{m}$, as measured by a spectrometer and refined by optimization. A typical spectrum of the light sources is provided in Appendix C, which can extract a peak wavelength of about $632 \mathrm{~nm}$ and a full width at half maximum (FWHM) of about $15 \mathrm{~nm}$. The images can be divided into two categories: high intensity "bright-field" data and "dark-field" images produced primarily by the scattered light. ${ }^{44}$ Taking into account the system configuration described above, the calculated overlap of image spectra is $78 \%{ }^{45}$

\subsubsection{Reconstruction methods}

A phase extraction algorithm is then applied to the images to reconstruct the transmission function $o(x, y)$ of the sample. The set of images holds not only phase information, but also highfrequency data, which give the reconstructed object high resolution and complex values.

We are looking for such an object $O\left(k_{x}, k_{y}\right)$ that would minimize the norm of the difference between the captured images $I_{l}(x, y), l=1, \ldots, L$, and the ones that would have been produced given the observation model of Eq. (6). The solution is sought in the Fourier space $\left[O\left(k_{x}, k_{y}\right)\right.$ rather than $o(x, y)]$ for convenience. It should be noted that different formulations are possible and that not all are posed as minimization problems with explicit cost functions. ${ }^{46} \mathrm{In}$ all cases, however, the nonconvex nature must be acknowledged. Many different approaches may be found in the literature. ${ }^{47}$

The most widespread method, which is a G-S type procedure, ${ }^{3}$ is used in the current work. The algorithm uses two nested loops to reconstruct the spectrum $O\left(k_{x}, k_{y}\right)$ of the object. The internal loop runs through all the data, with one image at a time. At each step $l$, a complex object is generated for the illumination angle of the l'th LED, based on the current estimation $O\left(k_{x}, k_{y}\right)$ and the imaging model. The amplitude component of this object is swapped with the square root of the captured image $\sqrt{I_{l}(x, y)}$. Then, the 2-D FT of the resulting complex image is used to 


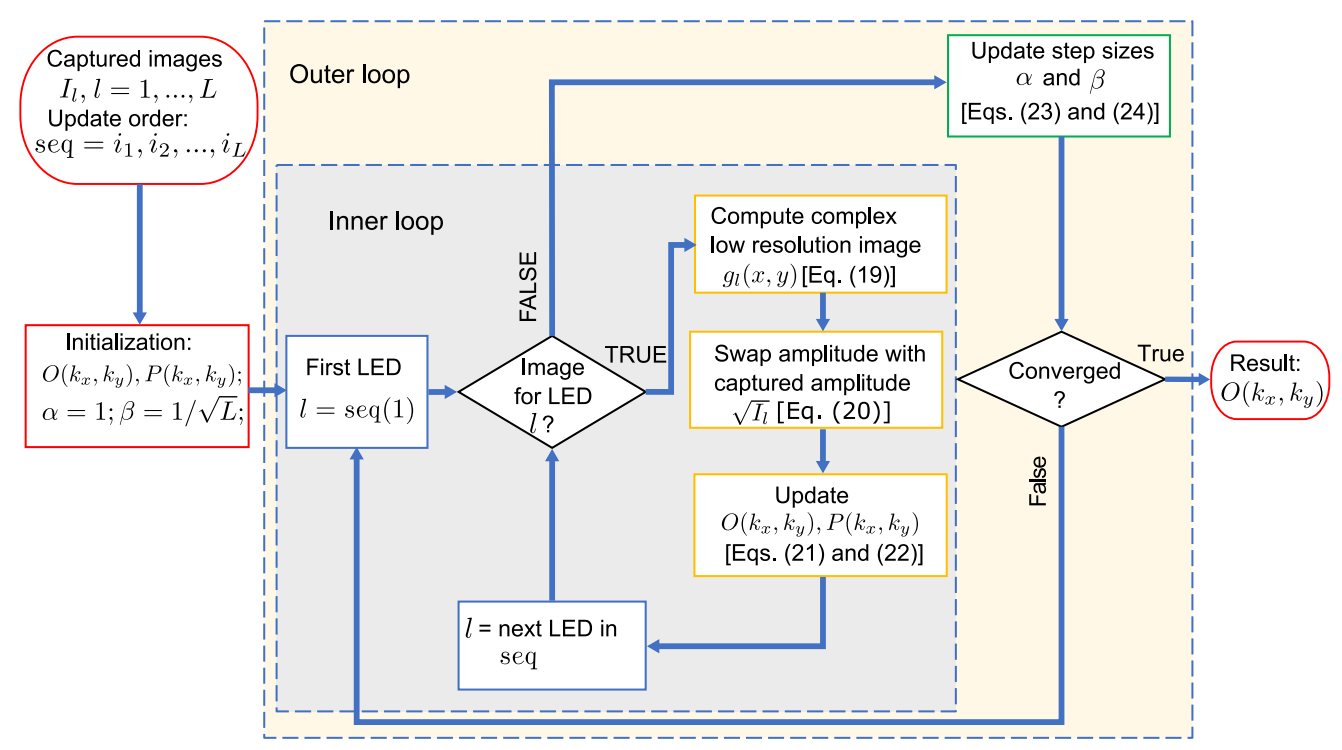

Fig. 4 Algorithm flowchart of reconstruction procedure for FPM. Equations are in Appendix B.

update the corresponding part of the estimated spectrum $O\left(k_{x}, k_{y}\right)$. The outer loop refines the result of the reconstruction.

Along with the reconstruction of the object $O\left(k_{x}, k_{y}\right)$ itself, we also apply a procedure for retrieving the pupil function $P\left(k_{x}, k_{y}\right)$ to correct the optical aberrations. ${ }^{48}$ This is a standard practice for the FPM procedure. In addition, to ensure greater convergence and robustness to noise, an adaptive step-size strategy is used. ${ }^{49}$ Lastly, or rather, first of all, the data are preprocessed to eliminate background offsets from raw images. ${ }^{50}$

The main workflow of the computer program is shown in Fig. 4. Convergence has been monitored using the two criteria that are commonly used for FPM. ${ }^{44}$ They are the amplitude cost (AC),

$$
\mathrm{AC}=\sum_{l} \sum_{x, y}\left\{\sqrt{\left[I_{l}(x, y)\right]_{\text {measured }}}-\sqrt{\left[I_{l}(x, y)\right]_{\text {simulated }}}\right\}^{2}
$$

and the convergence index (CI),

$$
\mathrm{CI}=\sum_{l} \frac{\text { mean }_{x, y}\left\{\sqrt{\left[I_{l}(x, y)\right]_{\text {simulated }}}\right\}}{\sum_{x, y}\left|\sqrt{\left[I_{l}(x, y)\right]_{\text {measured }}}-\sqrt{\left[I_{l}(x, y)\right]_{\text {simulated }}}\right|} .
$$

The behavior of both quantities as functions of the iteration index $t$ is shown in Fig. 9(c).

\section{Results and Analysis}

\subsection{LI-DHM Phase Retrieval Results}

The results from the G-S based algorithm are exposed and analyzed. The hologram captured by LI-DHM is shown in Fig. 5. The distance between the object plane and the sensor plane retrieved by the SOG autofocusing method is $17.881 \mathrm{~cm}$. The equivalent reconstruction distance using plane waves as reference waves is $99.987 \mathrm{~cm}$. To verify that autofocusing is reliable, the same procedure has been applied to two holograms that have been captured at two other axial positions translated by $100 \mu \mathrm{m}$. The figures provided by the SOG are 17.871 and $17.860 \mathrm{~cm}$, respectively. With a spacing of 90 and $110 \mu \mathrm{m}$, an error of $10 \mu \mathrm{m}$ over a distance of about $17.881 \mathrm{~cm}$ appears to be more than acceptable.

After ensuring such a reliability for the autofocusing technique, the iterative phase retrieval procedure has been launched. The reconstructed phase distribution is shown in Fig. 6(a). The 


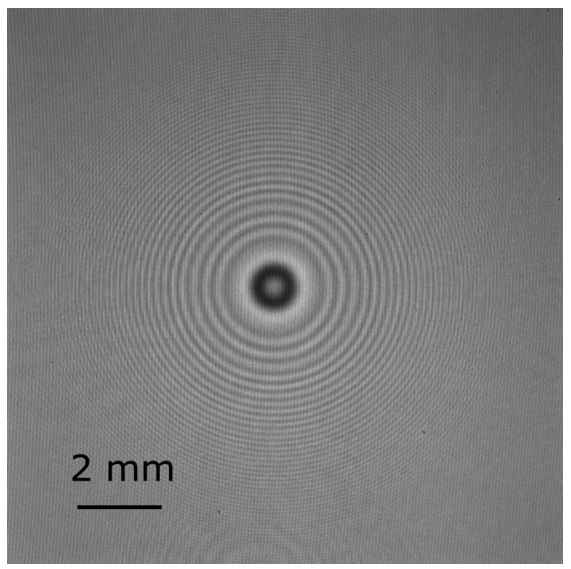

Fig. 5 Hologram of $146-\mu \mathrm{m}$ transparent glass microsphere captured by the LI-DHM instrument.

diameter of the round shape is $828.8 \mu \mathrm{m}$ with $5.59 \times$ magnification. Without magnification, such a value would drop to $148.3 \mu \mathrm{m}$, which is only $2 \mu \mathrm{m}$ larger than the diameter estimated with a phase-contrast microscope. In Fig. 6(b), the maximum phase shift $P_{\max }$ is clearly visible. It has been inferred to be about -10.10 rads. These outcomes reflect the QPI performances of the LI-DHM. It is worth mentioning that because of the restricted support introduced in the object domain (18 pixels larger than the object in radius), the algorithm converges with only 10 iterations.

The focus position and the maximum phase shift have then been used as an initial guess for the optimization process of the inverse problem. The minimum of the cost function has been found to be located at $\vec{p}=(17.878,-9.87)$. It means that the microsphere is $17.878 \mathrm{~cm}$ away from the camera, and the maximum phase shift it induces is -9.87 rads. Consistency with the result from the SOG autofocusing technique combined with the G-S phase retrieval method has been maintained.

As shown in Fig. 7, the cost function $\ell(\vec{p})$ exhibits a minimum at -9.87 rads. However, two other local minimums ( -25.88 and -18.36 rads) have been found as the maximum phase shift is scanned from -30 to -6 , with the object kept at $17.878 \mathrm{~cm}$. For those values of $P_{\max }$, three holograms were simulated for comparison purposes with the captured hologram. Sectioning the diffraction rings throughout their centers provided the fringes of Fig. 8. Great similarity between the experimental and the simulated curves can be observed. However, the oscillation featured for $P_{\max }=-9.87$ is closer to the ones captured by the camera in the very central area. It signifies that such a phase shift would probably be the one sought after.

(a)

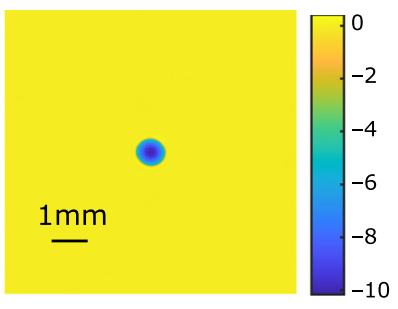

(b)

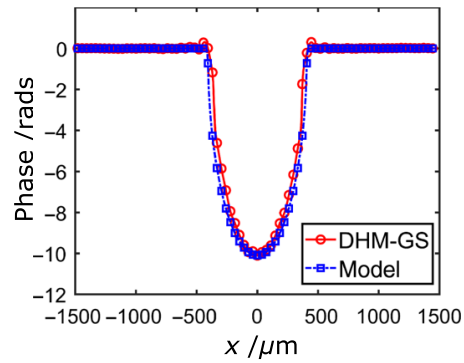

(c)

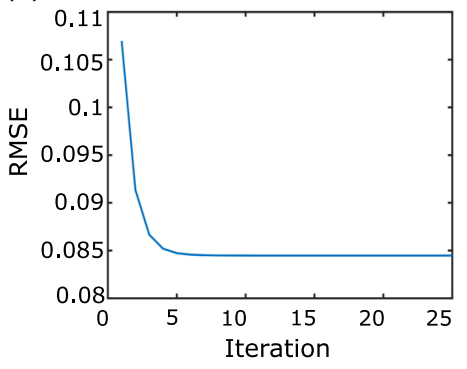

Fig. 6 Reconstruction of G-S-based iterative phase retrieval algorithm: (a) reconstructed phase distribution. (b) Curve with red circles is a scan through the center of (a). Curve with blue squares is theoretical phase distribution calculated according to Eq. (5), with magnified diameter of $816.1 \mu \mathrm{m}$ (measured diameter $146 \mu \mathrm{m}$ times magnification factor of 5.59) and maximum phase shift of -10.10 rads. (c) Root-mean-square error (RMSE) as a function of iteration number. Convergence has been attained after 10 iterations. 


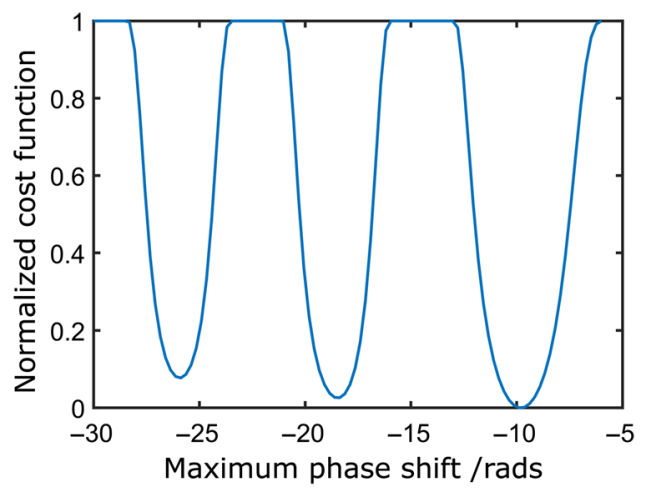

Fig. 7 Normalized cost function obtained by scanning maximum phase shift while keeping axial position at $17.878 \mathrm{~cm}$.
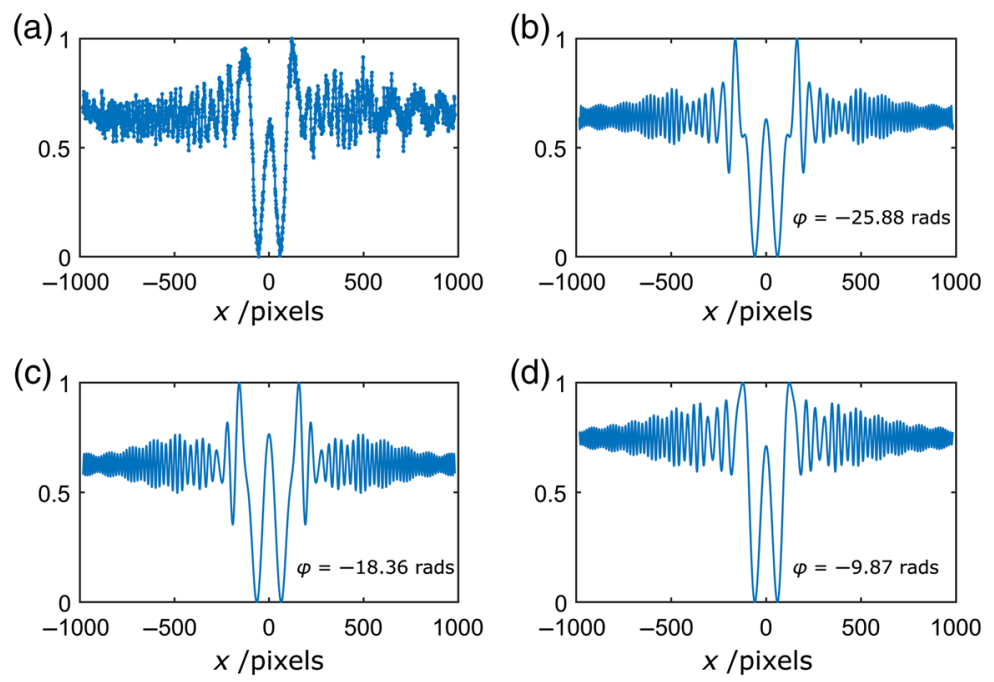

Fig. 8 Intensity distributions of diffraction fringes. (a) Profile of hologram captured by the LI-DHM. Profiles simulated for a distance object-camera set to $17.878 \mathrm{~cm}$ and a maximum phase shift equals to (b) $P_{\max }=-25.88$ rads, (c) $P_{\max }=-18.36$ rads, and (d) $P_{\max }=-9.87$ rads.

\subsection{FPM Phase Retrieval Result and Comparison}

Figure 9 shows phase retrieval results from FPM. The procedure has been executed patch by patch of $280 \times 280$ pixels each. The object has been enlarged by four times as compared to the original. With the measured magnification of 2.034 for the optical system and the camera's pixel pitch (PP) of $6.5 \mu \mathrm{m}$, the PP of a raw image is $3.196 \mu \mathrm{m}$. Consequently, the reconstructed image has a PP of 3.196/4 = $799 \mathrm{~nm}$. Those dimensions are taken in the object plane. The diameter of the phase disk is $148.6 \mu \mathrm{m}$ and the maximum phase shift is -8.3 rads (points with noncontinuous phase variation are neglected).

Phase curves obtained from the three methods (LI-DHM with G-S algorithm, LI-DHM with inverse problem approach, and FPM with G-S algorithm) are shown in Fig. 10. Good consistency between LI-DHM results may be observed. The results from both LI-DHM and FPM exhibit variations that follow Eq. (5) with diameter $148 \mu \mathrm{m}$ (radius $R=74 \mu \mathrm{m}$ ). However, here, FPM appears to underestimate the maximum phase shift by 2 rads. As the sample is optically thick, this result could be produced by an irrelevant approximation of a three-dimensional response by a 2-D function $o(x, y)$. The phase extent $P_{\max }$ associated with $o(x, y)$ may be estimated by integrating lightpaths within the object. Based on the axial index profile reported by Horstmeyer et al. ${ }^{20}$ [Fig. 4(a) therein], a value lower than the maximum phase extent could be obtained. The introduction of image formation model of Fourier ptychographic tomography would permit to test such a hypothesis. 
(a)

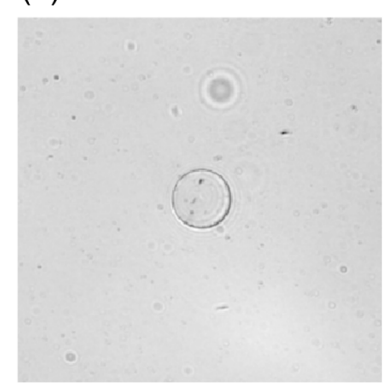

(d)

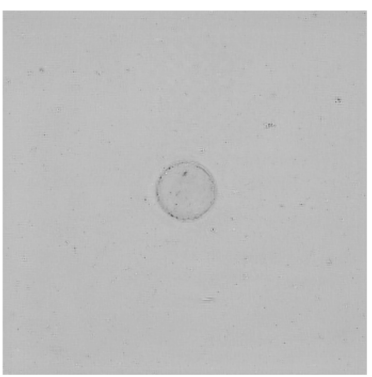

(b)

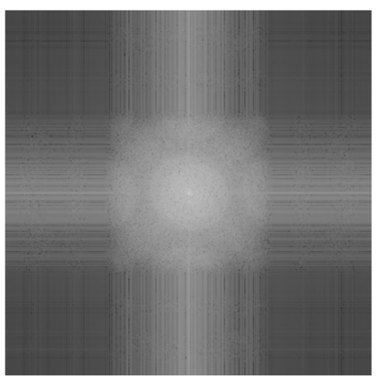

(e)

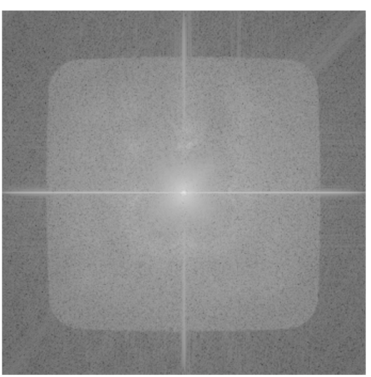

(c)

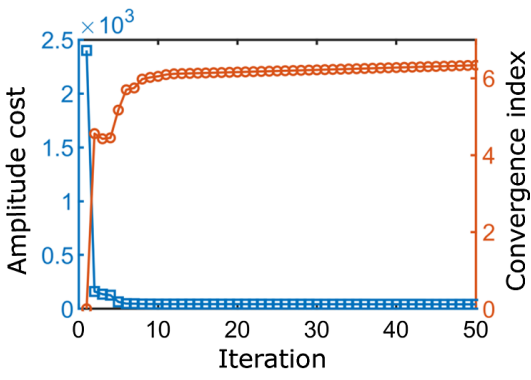

(f)

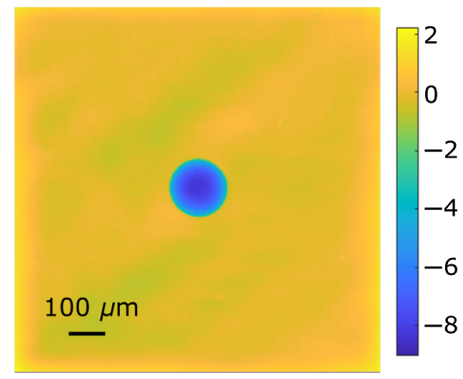

Fig. 9 Results from FPM. (a) Image captured as the center LED is switched on. (b) Amplitude spectrum of (a). (c) $\mathrm{AC}$ (blue squares) and $\mathrm{Cl}$ (red circles) as functions of iteration number. Convergence is attained when the former is minimized and the latter maximized. (d) Amplitude of reconstructed complex image. (e) Amplitude spectrum of (d) (logarithmic scale). (f) Reconstructed phase.

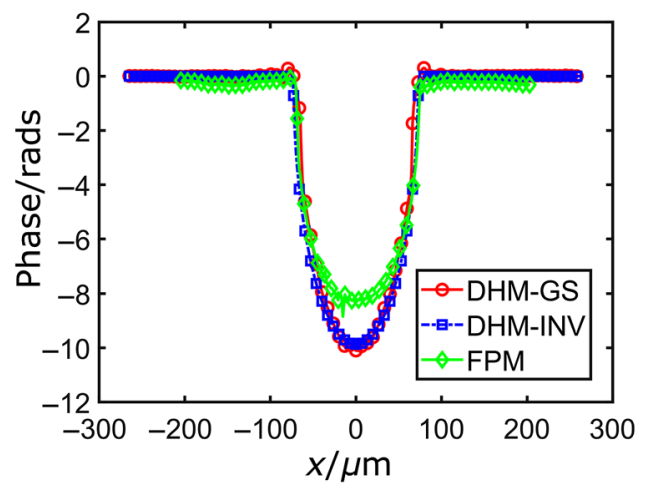

Fig. 10 Phase curves recovered by LI-DHM and FPM. Curve with red circles is obtained by LIDHM G-S algorithm. Curve with blue squares is phase distribution simulated according to Eq. (5), with diameter estimated from Fig. 1 and maximum phase shift provided by LI-DHM inverse problem approach. Curve with green diamonds represents phase retrieved from FPM G-S algorithm.

\section{Conclusion}

In this work, optical phase response from a large transparent object has been investigated with LI-DHM and compared to FPM. The sample is a 146- $\mu \mathrm{m}$ glass microsphere. A G-S type phase retrieval algorithm with positive absorption constraint has been used. A parameter-based inverse problem approach has also been applied to the same captured hologram. Reliability of the G-S recovered phase distribution has been assessed through focusing (object) position and maximum value of the phase shift induced by the sample. Analysis of LI-DHM and FPM maps reveals that both instruments are capable of providing phase distributions that would be expected from a microbead, confirming their QPI capability. FPM seems to provide smaller amplitudes as for the phase extent. Such an aspect will be studied in a forthcoming work. 


\section{Appendix A: Detailed Computation of the Cost Function $\ell(\overrightarrow{\boldsymbol{p}}, \boldsymbol{\alpha}, \boldsymbol{\beta})$}

The cost function provided by Eq. (4) will be exhibited here. We will use the same notations as in Ref. 41. We recall that $W(x, y)$ is a weighted mask that corresponds to the inverse of the hologram intensity variances at each pixel. $I_{H}(x, y)$ is the hologram intensity with the background subtracted. $I_{\text {simu }}(\vec{p} ; x, y)$ is the simulated hologram using Fresnel propagation algorithm. Here, we will use the shortened notation $I_{s}(\vec{p} ; x, y) . \alpha$ and $\beta$ are the variables that represent the absorption of the camera and the DC term of the hologram, respectively.

$$
\ell(\vec{p}, \alpha, \beta)=\sum_{x} \sum_{y} W(x, y) \cdot\left[I_{H}(x, y)-\alpha I_{s}(\vec{p} ; x, y)+\beta\right]^{2},
$$

$\ell(\vec{p}, \alpha, \beta)$ is minimized when $\beta=\beta^{*}$

$$
\left.\frac{d \ell(\vec{p}, \alpha, \beta)}{d \beta}\right|_{\beta=\beta^{*}}=0,
$$

which equals to

$$
2 \beta^{*} \sum_{x} \sum_{y} W(x, y)+2 \sum_{x} \sum_{y} W(x, y) I_{H}(x, y)-2 \alpha \sum_{x} \sum_{y} W(x, y) I_{s}(\vec{p} ; x, y)=0 .
$$

In the original development, $\sum_{x} \sum_{y} W(x, y) I_{H}(x, y)$ is skipped since $I_{H}$ has a zero mean. In our case, $W(x, y)$ may not be symmetric and $I_{H}$ is not necessary balanced because of the measurement noise. We need to keep all the terms

$$
\beta^{*}=\frac{\alpha \sum_{x} \sum_{y} W(x, y) I_{s}(\vec{p} ; x, y)-\sum_{x} \sum_{y} W(x, y) I_{H}(x, y)}{\sum_{x} \sum_{y} W(x, y)} .
$$

For the sake of clarity, we will adopt the same definitions as in Ref. 41.

$$
\begin{aligned}
\hat{S}_{W} & =\sum_{x} \sum_{y} W(x, y), \\
\hat{S}_{W I_{s}} & =\sum_{x} \sum_{y} W(x, y) I_{s}(\vec{p} ; x, y), \\
\hat{S}_{W I_{H}} & =\sum_{x} \sum_{y} W(x, y) I_{H}(x, y), \\
\hat{S}_{W I_{H} I_{s}} & =\sum_{x} \sum_{y} W(x, y) I_{H}(x, y) I_{s}(\vec{p} ; x, y), \\
\hat{S}_{W I_{s}^{2}} & =\sum_{x} \sum_{y} W(x, y)\left[I_{s}(\vec{p} ; x, y)\right]^{2}, \\
\hat{S}_{W I_{H}^{2}} & =\sum_{x} \sum_{y} W(x, y)\left[I_{H}(x, y)\right]^{2},
\end{aligned}
$$

where $\hat{S}$ represents $2-\mathrm{D}$ summation. With $\beta^{*}$ written as

$$
\beta^{*}=\frac{\alpha \hat{S}_{W I_{s}}-\hat{S}_{W I_{H}}}{\hat{S}_{W}}
$$

the cost function becomes

$$
\ell\left(\vec{p}, \alpha, \beta^{*}\right)=\sum_{x} \sum_{y} W(x, y) \cdot\left[I_{H}(x, y)-\alpha\left(I_{s}-\frac{\hat{S}_{W I_{s}}}{\hat{S}_{W}}\right)-\frac{\hat{S}_{W I_{H}}}{\hat{S}_{w}}\right]^{2},
$$


$\alpha^{*}$ will minimize $\ell(\vec{p}, \alpha, \beta)$ by imposing

$$
\left.\frac{d \ell\left(\vec{p}, \alpha, \beta^{*}\right)}{d \alpha}\right|_{\alpha=\alpha^{*}}=0,
$$

which entails

$$
\alpha^{*}=\frac{\hat{S}_{W I_{H} I_{s}}-\frac{\hat{S}_{W I_{H}} \hat{S}_{W I_{s}}}{\hat{S}_{W}}}{\hat{S}_{W I_{s}^{2}}-\frac{\hat{S}_{W I_{s}}^{2}}{\hat{S}_{W}}},
$$

where $\alpha$ represents the absorption of the sensor, it should be positive, $\alpha^{*}=\max \left(\alpha^{*}, 0\right)$. For optimal $\alpha$ and $\beta$, we obtain the expression of the cost function used in the algorithm as

$$
\begin{aligned}
& \ell\left(\vec{p}, \alpha^{*}, \beta^{*}\right)=\hat{S}_{W I_{H}^{2}}-\frac{\hat{S}_{W I_{H}}^{2}}{\hat{S}_{W}}-\frac{\left(\hat{S}_{W I_{H} I_{s}}-\frac{\hat{S}_{W I_{H}} \hat{S}_{W I_{s}}}{\hat{S}_{W}}\right)^{2}}{\hat{S}_{W I_{s}^{2}}-\frac{\hat{S}_{W I_{s}}^{2}}{\hat{S}_{W}}} \quad \alpha^{*}>0 \\
& =\hat{S}_{W I_{H}^{2}}-\frac{\hat{S}_{W I_{H}}^{2}}{\hat{S}_{W}} \quad \text { otherwise. }
\end{aligned}
$$

\section{Appendix B: Equations for Updating FPM Reconstruction}

We provide in this appendix the equations that were used to update the solution in the FP reconstruction procedure, at iteration $t$ of the outer loop (Fig. 4). For the sake of clarity, we set the

shifted FT to $O_{l}^{(t)}\left(k_{x}, k_{y}\right)=O^{(t)}\left(k_{x}-k_{x l}, k_{y}-k_{y l}\right)$, keeping in mind that we are aiming to retrieve $O\left(k_{x}, k_{y}\right)$ with the reconstruction procedure.

$$
\begin{gathered}
g_{l}^{(t)}(x, y)=\mathcal{F} \mathcal{T}^{-1}\left\{P^{(t)}\left(k_{x}, k_{y}\right) O_{l}^{(t)}\left(k_{x}, k_{y}\right)\right\}, \\
\tilde{g}_{l}^{(t)}(x, y)=\sqrt{\left(I_{l}\right)_{\text {measured }}} \exp \left(i \varphi_{l}^{(t)}\right) \quad \text { with } \varphi_{l}^{(t)}=\operatorname{angle}\left(g_{l}^{(t)}\right) .
\end{gathered}
$$

The notation $\mathcal{F} \mathcal{T}^{-1}\{$.$\} designates the inverse FT.$

$$
\begin{gathered}
O_{l}^{(t+1)}\left(k_{x}, k_{y}\right)=O_{l}^{(t)}\left(k_{x}, k_{y}\right) \\
+\alpha^{(t)} \frac{\left[P^{(t)}\right]^{*}\left(k_{x}, k_{y}\right)}{\left(\left|\left[P^{(t)}\right]^{*}\left(k_{x}, k_{y}\right)\right|^{2}\right)_{\max }}\left(\mathcal{F}\left\{\tilde{g}_{l}^{(t)}(x, y)\right\}-P^{(t)}\left(k_{x}, k_{y}\right) O_{l}^{(t)}\left(k_{x}, k_{y}\right)\right), \\
P^{(t+1)}\left(k_{x}, k_{y}\right)= \\
P^{(t)}\left(k_{x}, k_{y}\right) \\
+\beta^{(t)} \frac{\left[O_{l}^{(t)}\right]^{*}\left(k_{x}, k_{y}\right)}{\left(\left|\left[O_{l}^{(t)}\right]^{*}\left(k_{x}, k_{y}\right)\right|^{2}\right)_{\max }}\left(\mathcal{F}\left\{\tilde{g}_{l}^{(t)}(x, y)\right\}-P^{(t)}\left(k_{x}, k_{y}\right) O_{l}^{(t)}\left(k_{x}, k_{y}\right)\right) .
\end{gathered}
$$

The symbols $\mathcal{F}\{$.$\} and *$ represent the $\mathrm{FT}$ and the complex conjugate, respectively.

$$
\begin{gathered}
\alpha^{(t+1)}= \begin{cases}\frac{\alpha^{(t)}}{2} & \text { if } \frac{E^{(t-1)}-E^{(t)}}{E^{(t-1)}}<0.1 \\
\alpha^{(t)} & \text { otherwise }\end{cases} \\
\beta^{(t+1)}=\frac{\alpha^{(t+1)}}{\sqrt{L}} .
\end{gathered}
$$

For Eq. (23), we need to compute $E^{(s)}=\sum_{l} \sum_{x, y}\left(\left|g_{l}^{(s)}(x, y)\right|-\sqrt{\left[I_{l}(x, y)\right]_{\text {measured }}}\right)^{2}$, which is the AC at iteration $s$. 


\section{Appendix C: Additional Figures}

In this appendix, we supply an additional figure in response to the reviewers' comments. Figure 11(a) shows a typical spectral response of the red LEDs used for the FPM illumination. It has been measured by means of a spectrometer. A peak wavelength of $623 \mathrm{~nm}$ and a FWHM of $15 \mathrm{~nm}$ can be extracted. Figure 11(b) shows variations of TC as a function of axial translation. It has been computed from FPM captured images. The minimum at $\delta z=0 \mu \mathrm{m}$ identifies the best focus. The horizontal line that identifies the value $\left(\mathrm{TC}_{\max }+\mathrm{TC}_{\min }\right) / 2$ permits one to assess the width of the TC curve at its mean. A value of about $212 \mu \mathrm{m}$ has been obtained. At a fixed wavelength, the theoretical DOF depends only on the NA. ${ }^{27}$ It can be computed using the expression $\lambda /(\mathrm{NA})^{2}$. With $\lambda=630 \mathrm{~nm}$ and the manufacturer's NA of 0.055 , the estimated DOF is $208 \mu \mathrm{m}$. On the other hand, if the NA of 0.070, as estimated experimentally, is used, the DOF reduces to $129 \mu \mathrm{m}$.
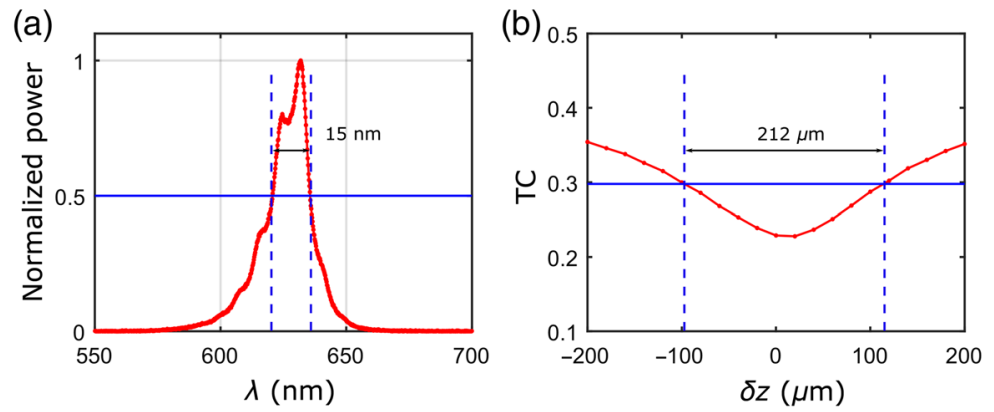

Fig. 11 (a) Typical spectrum of the illumination LEDs for FPM as measured by a spectrometer. The peak wavelength is $632 \mathrm{~nm}$ and the FWHM is $15 \mathrm{~nm}$. (b) TC as computed from FPM captured images. The curve exhibits a minimum at focus. The DOF as estimated by the width at mean is equal to $212 \mu \mathrm{m}$.

\section{Acknowledgments}

H. Li acknowledges the University of Strasbourg for his PhD scholarship that made this work possible. The authors are grateful to the anonymous reviewers for providing suggestions that helped to improve the quality of the paper. Appendix $\mathrm{C}$ has been added in response to their comments.

\section{References}

1. Y. Park, C. Depeursinge, and G. Popescu, "Quantitative phase imaging in biomedicine," Nat. Photonics 12(10), 578-589 (2018).

2. C. Zuo et al., "Transport of intensity equation: a tutorial," Opt. Laser Eng. 106187 (2020).

3. G. Zheng, R. Horstmeyer, and C. Yang, "Wide-field, high-resolution Fourier ptychographic microscopy," Nat. Photonics 7(9), 739 (2013).

4. D. Gabor, "A new microscopic principle," Nature 161, 777-778 (1948).

5. L. Denis et al., "Twin-image noise reduction by phase retrieval in in-line digital holography," Proc. SPIE 5914, 59140J (2005).

6. T. Latychevskaia and H.-W. Fink, "Solution to the twin image problem in holography," Phys. Rev. Lett. 98(23), 233901 (2007).

7. H. Sun et al., "Underwater digital holography for studies of marine plankton," Philos. Trans. R. Soc. A 366(1871), 1789-1806 (2008).

8. J. Zhang et al., "Adaptive pixel-super-resolved lensfree in-line digital holography for widefield on-chip microscopy," Sci. Rep. 7(1), 11777 (2017).

9. Y. Wu and A. Ozcan, "Lensless digital holographic microscopy and its applications in biomedicine and environmental monitoring," Methods 136, 4-16 (2018). 
10. O. Mudanyali, W. Bishara, and A. Ozcan, "Lensfree super-resolution holographic microscopy using wetting films on a chip," Opt. Express 19(18), 17378-17389 (2011).

11. T. W. Su et al., "Multi-angle lensless digital holography for depth resolved imaging on a chip," Opt. Express 18(9), 9690-9711 (2010).

12. W. Bishara et al., "Holographic pixel super-resolution in portable lensless on-chip microscopy using a fiber-optic array," Lab Chip 11(7), 1276-1279 (2011).

13. M. U. Daloglu et al., "Low-cost and portable UV holographic microscope for high-contrast protein crystal imaging," APL Photonics 4(3), 030804 (2019).

14. A. C. Sobieranski et al., "Portable lensless wide-field microscopy imaging platform based on digital inline holography and multi-frame pixel super-resolution," Light Sci. Appl. 4(10), e346 (2015).

15. V. Poher et al., "Lensfree in-line holographic detection of bacteria," Proc. SPIE 8086, 808619 (2011).

16. X. Ou et al., "Quantitative phase imaging via Fourier ptychographic microscopy," Opt. Lett. 38(22), 4845-4848 (2013).

17. A. J. Williams et al., "Fourier ptychographic microscopy for filtration-based circulating tumor cell enumeration and analysis," J. Biomed. Opt. 19(6), 066007 (2014).

18. R. Horstmeyer et al., "Digital pathology with Fourier ptychography," Comput. Med. Imaging Graphics 42, 38-43 (2015).

19. T. Nguyen et al., "Deep learning approach for Fourier ptychography microscopy," Opt. Express 26, 26470-26484 (2018).

20. R. Horstmeyer et al., "Diffraction tomography with Fourier ptychography," Optica 3(8), $827-835$ (2016).

21. J. Sun et al., "Single-shot quantitative phase microscopy based on color-multiplexed Fourier ptychography," Opt. Lett. 43(14), 3365-3368 (2018).

22. M. Stockmar et al., "Near-field ptychography: phase retrieval for inline holography using a structured illumination," Sci. Rep. 3, 1927 (2013).

23. M. H. Jericho et al., "Quantitative phase and refractive index measurements with point-source digital in-line holographic microscopy," Appl. Opt. 51(10), 1503-1515 (2012).

24. D. Gabor, "Microscopy by reconstructed wave-fronts," Proc. R. Soc. London A 197, 454-487 (1949).

25. J. Garcia-Sucerquia et al., "Digital in-line holographic microscopy," Appl. Opt. 45(5), 836-850 (2006).

26. J. P. Ryle, J. T. Sheridan, and S. McDonnell, "Lensless multispectral digital in-line holographic microscope," J. Biomed. Opt. 16(12), 126004 (2011).

27. C. P. Shillaber, Photomicrography in Theory and Practice, John Wiley \& Sons, Inc., New York (1959).

28. R. W. Gerchberg and W. O. Saxon, "A practical algorithm for the determination of phase from image and diffraction plane pictures," Optik 35, 237-246 (1972).

29. C. Fournier et al., "Inverse problem approaches for digital hologram reconstruction," Proc. SPIE 8043, 80430S (2011).

30. T. Shimobaba and T. Ito, Computer Holography: Acceleration Algorithms and Hardware Implementations, CRC Press, Boca Raton, Florida (2019).

31. Y. Zhang et al., "Edge sparsity criterion for robust holographic autofocusing," Opt. Lett. 42(19), 3824-3827 (2017).

32. M. Tamamitsu et al., "A robust holographic autofocusing criterion based on edge sparsity: comparison of Gini index and Tamura coefficient for holographic autofocusing based on the edge sparsity of the complex optical wavefront," in Quantitative Phase Imaging IV, G. Popescu and Y. Park, Eds., Vol. 10503, pp. 22-31 (2018).

33. T. Latychevskaia and H.-W. Fink, "Reconstruction of purely absorbing, absorbing and phase-shifting, and strong phase-shifting objects from their single-shot in-line holograms," Appl. Opt. 54(13), 3925-3932 (2015).

34. J. Hu, Q. Li, and Y. Zhou, "Support-domain constrained phase retrieval algorithms in terahertz in-line digital holography reconstruction of a nonisolated amplitude object," Appl. Opt. 55(2), 379-386 (2016). 
35. K. M. Molony et al., "Reconstruction algorithms applied to in-line Gabor digital holographic microscopy," Opt. Commun. 283(6), 903-909 (2010).

36. W. Qu et al., "Recording and reconstruction of digital Gabor hologram," Optik 121(23), 2179-2184 (2010).

37. T. Latychevskaia and H.-W. Fink, "Practical algorithms for simulation and reconstruction of digital in-line holograms," Appl. Opt. 54(9), 2424-2434 (2015).

38. K. Matsushima and T. Shimobaba, "Band-limited angular spectrum method for numerical simulation of free-space propagation in far and near fields," Opt. Express 17(22), 19662-19673 (2009).

39. A. Berdeu et al., "Reconstruction of in-line holograms: combining model-based and regularized inversion," Opt. Express 27(10), 14951-14968 (2019).

40. F. Soulez et al., "Inverse-problem approach for particle digital holography: accurate location based on local optimization," J. Opt. Soc. Am. A 24(4), 1164-1171 (2007).

41. L. Denis, "Traitement et analyse quantitative d'hologrammes numériques," PhD Thesis, Saint-Etienne (2006).

42. J. Dohet-Eraly et al., "Analysis of three-dimensional objects in quantitative phase contrast microscopy: a validity study of the planar approximation for spherical particles," Proc. SPIE 11351, 113510Y (2020).

43. M. J. Best, Quadratic Programming with Computer Programs, Chapman and Hall/CRC, Boca Raton, Florida (2017).

44. G. Zheng, Fourier Ptychographic Imaging: A MATLAB Tutorial, Morgan \& Claypool Publishers, San Rafael, California (2016).

45. J. Sun et al., "Sampling criteria for Fourier ptychographic microscopy in object space and frequency space," Opt. Express 24(14), 15765-15781 (2016).

46. A. Konijnenberg, W. Coene, and H. Urbach, "Study of cost functionals for ptychographic phase retrieval to improve the robustness against noise, and a proposal for another noiserobust ptychographic phase retrieval scheme," arXiv:1703.10892 (2017).

47. L.-H. Yeh, "Analysis and comparison of Fourier ptychographic phase retrieval algorithms," Master's Thesis, EECS Department, University of California, Berkeley, California (2016).

48. X. Ou, G. Zheng, and C. Yang, "Embedded pupil function recovery for Fourier ptychographic microscopy," Opt. Express 22(5), 4960-4972 (2014).

49. C. Zuo, J. Sun, and Q. Chen, "Adaptive step-size strategy for noise-robust Fourier ptychographic microscopy," Opt. Express 24(18), 20724-20744 (2016).

50. Y. Zhang et al., "Data preprocessing methods for robust Fourier ptychographic microscopy," Opt. Eng. 56(12), 123107 (2017).

Hongyu Li is a PhD student at the University of Strasbourg. He received his BS degree in optoelectronics engineering from Huazhong University of Science and Technology in 2016 and his MS degree in nanophotonic from the University of Strasbourg in 2018. He is currently working on his thesis about digital holography and surface plasmonic resonance.

Anastasia Bozhok is a PhD student at the University of Strasbourg. She received her specialist degree from the Far Eastern Federal University in Russia and her master's degree from the Grenoble Institute of Technology in France. She is currently working in the field of computational imaging.

Yoshitate Takakura is currently an associate professor at the University of Strasbourg, France. He lectures digital signal processing, spectral analysis and physics-related topics such as plasmonics. He is involved in developing optical imaging techniques that include modelization, instrumentation, and processing.

Jean Dellinger is an associate professor at the University of Strasbourg. His research activities focus on development of multimodal imaging system. This includes FPM, plenoptic microscopy, and polarimetric systems (Mueller polarimeter).

Patrice Twardowski received his master's degree in applied physics from the University of Metz, France, in 1983, his engineer master's degree from Ecole Nationale Supérieure de 
Physique de Strasbourg in 1985, and his $\mathrm{PhD}$ in optics and photonics from Louis Pasteur University, Strasbourg, France, in 1990. In 1991, he joined the University of Strasbourg and became an associate professor. His research interests include diffractive optical elements, phase measurement, and optical design.

Jihad Zallat is a professor at the University of Strasbourg. He heads the research group in Remote Sensing, Radiometry and Optical Imaging of the ICube laboratory. His research area includes imagery, photonics, and systems. 